\title{
Treating dyslipidemia of the metabolic syndrome: where's the evidence?
}

\author{
Robert H Eckel
}

The metabolic syndrome affects $24 \%$ of adults in the US, ${ }^{1}$ and physicians are increasingly looking beyond the control of LDL cholesterol to reduce such individuals' risk for cardiovascular disease and type 2 diabetes. Intervention in these patients starts with lifestyle modification: improved diet and increased physical activity can result in $\sim 10 \%$ weight loss that favorably modifies most criteria of the metabolic syndrome (e.g. waist circumference, blood pressure, HDL cholesterol, triglyceride and glucose levels). If lifestyle changes alone fail, pharmacological management of the residual risk is attempted.

For serum triglycerides the current target is $150 \mathrm{mg} / \mathrm{dl}(1.7 \mathrm{mmol} / \mathrm{l})$; for HDL cholesterol, levels of $>40 \mathrm{mg} / \mathrm{dll}(1.02 \mathrm{mmol} / \mathrm{l})$ for men and $>50 \mathrm{mg} / \mathrm{dl}(1.28 \mathrm{mmol} / \mathrm{l})$ for women are recommended. Despite substantial evidence that HDLcholesterol levels and fasting triglyceride levels are related to cardiovascular risk, no convincing evidence exists that achieving these target levels has benefits beyond improvements in other risk factors, particularly in LDL cholesterol.

Trials of agents that target triglycerides (e.g. fibrates and omega-3 fatty acids) demonstrated variable cardiovascular risk reduction, with little evidence that reduced triglyceride levels explain this benefit. ${ }^{2}$ The Veterans Affairs HighDensity Lipoprotein Intervention Trial (VA-HIT), in which LDL cholesterol levels were identical in the treated and placebo groups, is exemplary: despite the benefits of gemfibrozil in men (particularly in relation to insulin resistance and type 2 diabetes), ${ }^{2}$ the observed amount of triglyceride lowering failed to explain this benefit of fibrate therapy. ${ }^{3}$

Ideal goals for HDL-cholesterol levels also remain unclear. Niacin remains the best drug to raise HDL cholesterol levels; the safety and benefit of cholesteryl ester transfer protein inhibitors is uncertain. The Coronary Drug Project identified benefits of niacin therapy, albeit mostly in the open-label phase, ${ }^{4}$ and HDL-cholesterol levels were measured in only a minority of participants. The Arterial Biology
For patients

with low HDLcholesterol

levels, we

should first

focus on LDL

cholesterol,

and perhaps

introduce even

lower targets...

RH Eckel is Professor

of Medicine in

the Division of

Endocrinology,

Metabolism and

Diabetes and

the Division

of Cardiology,

and Charles A

Boettcher II Chair in

Atherosclerosis, at the

University of Colorado

at Denver and Health

Sciences Center, and

Director of the Lipid

Clinic at University

Hospital Denver, CO,

USA.

\section{Competing interests}

The author declared he has

no competing interests.

www.nature.com/clinicalpractice doi:10.1038/ncpendmet0514 for the Investigation of the Treatment Effects of Reducing Cholesterol 2 (ARBITER-2) trial demonstrated a borderline $(P<0.1)$ lack of progress in carotid intima-media thickness when extended-release niacin was added to statin therapy; HDL cholesterol was higher and triglyceride levels were lower in the niacin-treated group. ${ }^{5}$

So where does that leave the practitioner's therapeutic decision-making for patients with dyslipidemia, with or without the metabolic syndrome? For fasting hypertriglyceridemia, perhaps levels of apolipoprotein B (ApoB $)^{6}$ and/or LDL particle number ${ }^{7}$ will help to determine which patients should be treated, and to target the appropriate variables. Control of ApoB is expensive, and non-HDL cholesterol might be equally predictive and cheaper to control. The strong relationship between non-HDL cholesterol and ApoB levels in large populations might not, however, predict the atherogenic burden of a given individual. Further insight is clearly needed.

For patients with low HDL-cholesterol levels, we should first focus on LDL cholesterol, and perhaps introduce even lower targets for LDL cholesterol, before HDL cholesterol levels are treated with drugs. Evidence from both primary and secondary prevention trials suggests that the lower the LDL cholesterol, the better. ${ }^{2}$ In my practice, HDL cholesterol levels below $30 \mathrm{mg} / \mathrm{dl}(0.77 \mathrm{mmol} / \mathrm{l})$ translate to an LDL cholesterol goal of $15 \mathrm{mg} / \mathrm{dl}(0.38 \mathrm{mmol} / \mathrm{l})-$ lower than that determined by present guidelines. Not until clinical trials substantiate an independent benefit for HDL-raising therapy will I institute HDL-cholesterol-raising therapies before LDL cholesterol is substantially reduced. For dyslipidemia of the metabolic syndrome, let's get the LDL down! ${ }^{8}$

Supplementary information, in the form of a list of references cited in this article, is available on the Nature Clinical Practice Endocrinology \& Metabolism website. 